\title{
ON FURTHER STRENGTHENED HARDY-HILBERT'S INEQUALITY
}

\section{LÜ ZHONGXUE}

\author{
Received 7 May 2002
}

We obtain an inequality for the weight coefficient $\omega(q, n)(q>1,1 / p+1 / q=1, n \in \mathbb{N})$ in the form $\omega(q, n)=: \sum_{m=1}^{\infty}(1 /(m+n))(n / m)^{1 / q}<\pi / \sin (\pi / p)-1 /\left(2 n^{1 / p}+(2 / a) n^{-1 / q}\right)$ where $0<a<147 / 45$, as $n \geq 3$; $0<a<(1-C) /(2 C-1)$, as $n=1,2$, and $C$ is an Euler constant. We show a generalization and improvement of Hilbert's inequalities. The results of the paper by Yang and Debnath are improved.

2000 Mathematics Subject Classification: 26D15.

1. Introduction. The following inequalities are well known as Hardy-Hilbert's inequalities:

$$
\begin{gathered}
\sum_{m=1}^{\infty} \sum_{n=1}^{\infty} \frac{a_{m} b_{n}}{m+n}<\frac{\pi}{\sin (\pi / p)}\left(\sum_{n=1}^{\infty} a_{n}^{p}\right)^{1 / p}\left(\sum_{n=1}^{\infty} b_{n}^{q}\right)^{1 / q}, \\
\sum_{n=1}^{\infty}\left(\sum_{m=1}^{\infty} \frac{a_{m}}{m+n}\right)^{p}<\left(\frac{\pi}{\sin (\pi / p)}\right)^{p} \sum_{n=1}^{\infty} a_{n}^{p} .
\end{gathered}
$$

In recent years, Gao [1, 2], Xu and Guo [4], Hsu and Wang [3], Yang [6], and Yang and Gao [7] gave some distinct improvements of (1.1). Yang and Debnath [5] gave a strengthened version by the following inequality:

$$
\omega(q, n)=: \sum_{m=1}^{\infty} \frac{1}{m+n}\left(\frac{n}{m}\right)^{1 / q}<\frac{\pi}{\sin (\pi / p)}-\frac{1}{2 n^{1 / p}+n^{-1 / q}} .
$$

In this paper, we show a new generalization and improvement of (1.1) by improving (1.3).

First we introduce some lemmas.

LEMMA 1.1 [2]. Let $f(x)>0, f^{(2 r-1)}(x)<0, f^{(2 r)}(x) \geq 0, x \in[1, \infty), r=1,2$, $f^{(r)}(\infty)=0, r=0,1,2,3,4$, and $\int_{1}^{\infty} f(x) d x<\infty$, then

$$
\sum_{m=1}^{\infty} f(m) \leq \int_{1}^{\infty} f(x) d x+\frac{1}{2} f(1)-\frac{1}{12} f^{\prime}(1) .
$$

LEMMA 1.2 [5]. Let $q>1,1 / p+1 / q=1, n \in \mathbb{N}$, then

$$
\omega(q, n)<\frac{\pi}{\sin (\pi / p)}-\frac{1}{n^{1 / p}}\left[f_{n}(p)+g_{n}(p)\right],
$$


where $\omega(q, n)$ is defined by (1.3), and

$$
\begin{aligned}
& f_{n}(p):=p+\frac{1}{12 p}+\frac{1}{(1+p) n}+\frac{1}{12 p n^{2}}+\frac{1}{3(1+3 p) n^{3}} \\
& g_{n}(p):=-\frac{1}{12 p n}-\frac{1}{2(1+2 p) n^{2}}-\frac{7}{12}-\frac{1}{2 n}+\frac{1}{12 n^{2}}-\frac{7}{12 n^{3}} .
\end{aligned}
$$

LEMMA 1.3 [5]. Let $p>1, n \in \mathbb{N}$, then

$$
f_{n}(p)+g_{n}(p)>\frac{1}{2}-\frac{1}{12 n}-\frac{1}{2 n^{3}}
$$

LEMMA 1.4. Let $q>1,1 / p+1 / q=1, n \in \mathbb{N}$, then

$$
\begin{aligned}
& \omega(q, n)=: \sum_{m=1}^{\infty} \frac{1}{m+n}\left(\frac{n}{m}\right)^{1 / q}<\frac{\pi}{\sin (\pi / p)}-\frac{1}{2 n^{1 / p}+(2 / a) n^{-1 / q}}, \\
& \omega(p, n)=: \sum_{m=1}^{\infty} \frac{1}{m+n}\left(\frac{n}{m}\right)^{1 / p}<\frac{\pi}{\sin (\pi / p)}-\frac{1}{2 n^{1 / q}+(2 / a) n^{-1 / p}}
\end{aligned}
$$

where $0<a<147 / 45$ as $n \geq 3 ; 0<a<(1-C) /(2 C-1)$ as $n=1,2$, and $C$ is an Euler constant.

ProOF. For $n \geq 3$,

$$
\left(\frac{1}{2}-\frac{1}{12 n}-\frac{1}{2 n^{3}}\right)\left(x+\frac{1}{n y}\right)=\frac{x}{2}+\frac{1}{n}\left(\frac{1}{2 y}-\frac{x}{12}-\frac{1}{12 y n}-\frac{x}{2 n^{2}}-\frac{1}{2 y n^{3}}\right),
$$

where $x>0, y>0, x y=a$.

We first prove

$$
\frac{1}{2 y}-\frac{x}{12}-\frac{1}{12 y n}-\frac{x}{2 n^{2}}-\frac{1}{2 y n^{3}}=\frac{6 n^{3}-x y n^{3}-n^{2}-6 x y n-6}{12 y n^{3}}>0 .
$$

Formula (1.11) is equivalent to $\psi(n)=6 n^{3}-x y n^{3}-n^{2}-6 x y n-6>0$.

Since $\psi^{\prime}(x)=18 x^{2}-3 a x^{2}-2 x-6 a, \psi^{\prime \prime}(x)=36 x-6 a x-2$. When $0<a<147 / 45$, $\psi^{\prime \prime}(x)=36 x-6 a x-2>0, \psi^{\prime}(3)=156-33 a>0$, then $\psi^{\prime}(x)>0$ and $\psi(3)=147-$ $45 a>0$, hence $\psi(n)>0$ for $n \geq 3$. Therefore $\left(1 / 2-1 / 12 n-1 / 2 n^{3}\right)(x+1 / n y)>x / 2$. Namely, $1 / 2-1 / 12 n-1 / 2 n^{3}>1 /\left(2+2(\text { an })^{-1}\right)$, for $n \geq 3$. By (1.5) and (1.7), we have

$$
\begin{aligned}
\omega(q, n) & <\frac{\pi}{\sin (\pi / p)}-\frac{1}{n^{1 / p}}\left(\frac{1}{2}-\frac{1}{12 n}-\frac{1}{2 n^{3}}\right) \\
& <\frac{\pi}{\sin (\pi / p)}-\frac{1}{2 n^{1 / p}+(2 / a) n^{-1 / q}} .
\end{aligned}
$$


Since $\phi(x)=1 /\left(2 x+2(x n)^{-1}\right)$ is strictly increasing on $(0, \infty)$ and $0<a<(1-$ $C) /(2 C-1)$, by $\omega(q, n)<\pi / \sin (\pi / p)-(1-C) / n^{1 / p}$ (see [7]), we have, when $n=1$,

$$
\begin{aligned}
\omega(q, 1) & <\frac{\pi}{\sin (\pi / p)}-\frac{1-C}{1}<\frac{\pi}{\sin (\pi / p)}-\frac{1}{2+2(2 C-1) /(1-C)} \\
& \leq \frac{\pi}{\sin (\pi / p)}-\frac{1}{2+2 / a} ;
\end{aligned}
$$

when $n=2$,

$$
\omega(q, 2)<\frac{\pi}{\sin (\pi / p)}-\frac{1-C}{2^{1 / p}}<\frac{\pi}{\sin (\pi / p)}-\frac{1}{2^{1 / p}} \frac{1}{2+(2 / a)(1 / 2)}
$$

By (1.12), (1.13), and (1.14), (1.8) is valid for any $n \in \mathbb{N}$. Interchanging $p, q$ in (1.8), since $\pi / \sin (\pi / p)=\pi / \sin (\pi / q)$, we have (1.9). The lemma is proved.

2. Main results. Now we introduce main results.

THEOREM 2.1. Let $p>1,1 / p+1 / q=1, a_{n} \geq 0, b_{n} \geq 0,0<\sum_{n=1}^{\infty} a_{n}^{p}<\infty$, and $0<\sum_{n=1}^{\infty} b_{n}^{q}<\infty$. Then

$$
\begin{gathered}
\sum_{m=1}^{\infty} \sum_{n=1}^{\infty} \frac{a_{m} b_{n}}{m+n} \\
<\left(\left(\frac{\pi}{\sin (\pi / p)}-\frac{b}{2+2 b}\right) a_{1}^{p}+\left(\frac{\pi}{\sin (\pi / p)}-\frac{b}{2^{1 / p}(2 b+2)}\right) a_{2}^{p}\right. \\
\left.+\sum_{n=3}^{\infty}\left(\frac{\pi}{\sin (\pi / p)}-\frac{1}{2 n^{1 / p}+(2 / a) n^{-1 / q}}\right) a_{n}^{p}\right)^{1 / p} \\
\times\left(\left(\frac{\pi}{\sin (\pi / p)}-\frac{b}{2+2 b}\right) b_{1}^{q}+\left(\frac{\pi}{\sin (\pi / p)}-\frac{b}{2^{1 / q}(2 b+2)}\right) b_{2}^{q}\right. \\
\left.+\sum_{n=3}^{\infty}\left(\frac{\pi}{\sin (\pi / p)}-\frac{1}{2 n^{1 / q}+(2 / a) n^{-1 / p}}\right) b_{n}^{q}\right)^{1 / q}, \\
\sum_{m=1}^{\infty}\left(\sum_{n=1}^{\infty} \frac{a_{n}}{m+n}\right)^{p} \\
<\left(\frac{\pi}{\sin (\pi / p)}\right)^{p-1}\left(\left(\frac{\pi}{\sin (\pi / p)}-\frac{b}{2+2 b}\right) a_{1}^{p}+\left(\frac{\pi}{\sin (\pi / p)}-\frac{b^{1 / p}(2 b+2)}{2}\right) a_{2}^{p}\right. \\
\left.+\sum_{n=3}^{\infty}\left(\frac{\pi}{\sin (\pi / p)}-\frac{1}{2 n^{1 / p}+(2 / a) n^{-1 / q}}\right) a_{n}^{p}\right)
\end{gathered}
$$

where $0<a<147 / 45,0<b<(1-C) /(2 C-1)$. 
In particular, when $a=b=e, e$ is a constant,

$$
\begin{aligned}
\sum_{m=1}^{\infty} \sum_{n=1}^{\infty} \frac{a_{m} b_{n}}{m+n}< & \left(\sum_{n=1}^{\infty}\left(\frac{\pi}{\sin (\pi / p)}-\frac{1}{2 n^{1 / p}+(2 / e) n^{-1 / q}}\right) a_{n}^{p}\right)^{1 / p} \\
& \times\left(\sum_{n=1}^{\infty}\left(\frac{\pi}{\sin (\pi / p)}-\frac{1}{2 n^{1 / q}+(2 / e) n^{-1 / p}}\right) b_{n}^{q}\right)^{1 / q}, \\
\sum_{m=1}^{\infty}\left(\sum_{n=1}^{\infty} \frac{a_{n}}{m+n}\right)^{p}< & \left(\frac{\pi}{\sin (\pi / p)}\right)^{p-1} \sum_{n=1}^{\infty}\left(\frac{\pi}{\sin (\pi / p)}-\frac{1}{2 n^{1 / p}+(2 / e) n^{-1 / q}}\right) a_{n}^{p} .
\end{aligned}
$$

Proof. By Hölder's inequality, we have

$$
\begin{aligned}
\sum_{m=1}^{\infty} \sum_{n=1}^{\infty} \frac{a_{m} b_{n}}{m+n} & =\sum_{m=1}^{\infty} \sum_{n=1}^{\infty} \frac{a_{m}}{(m+n)^{1 / p}}\left(\frac{m}{n}\right)^{1 / p q} \frac{b_{n}}{(m+n)^{1 / q}}\left(\frac{n}{m}\right)^{1 / p q} \\
& \leq\left(\sum_{m=1}^{\infty} \sum_{n=1}^{\infty} \frac{a_{m}^{p}}{m+n}\left(\frac{m}{n}\right)^{1 / q}\right)^{1 / p}\left(\sum_{m=1}^{\infty} \sum_{n=1}^{\infty} \frac{b_{n}^{q}}{m+n}\left(\frac{n}{m}\right)^{1 / p}\right)^{1 / q} \\
& =\left(\sum_{n=1}^{\infty} \omega(q, n) a_{n}^{p}\right)^{1 / p}\left(\sum_{n=1}^{\infty} \omega(p, n) b_{n}^{q}\right)^{1 / q} .
\end{aligned}
$$

By (1.8) and (1.9), (2.1) is valid.

By Hölder's inequality and (1.9), we have

$$
\begin{aligned}
\sum_{n=1}^{\infty} \frac{a_{m}}{m+n} & =\sum_{n=1}^{\infty} \frac{a_{n}}{(m+n)^{1 / p}}\left(\frac{n}{m}\right)^{1 / p q} \frac{1}{(m+n)^{1 / q}}\left(\frac{m}{n}\right)^{1 / p q} \\
& \leq\left(\sum_{n=1}^{\infty} \frac{a_{n}^{p}}{m+n}\left(\frac{m}{n}\right)^{1 / q}\right)^{1 / p}\left(\sum_{n=1}^{\infty} \frac{1}{m+n}\left(\frac{m}{n}\right)^{1 / p}\right)^{1 / q} \\
& <\left(\frac{\pi}{\sin (\pi / p)}\right)^{1 / q}\left(\sum_{n=1}^{\infty} \frac{a_{n}^{p}}{m+n}\left(\frac{n}{m}\right)^{(2-\lambda) / q}\right)^{1 / p} .
\end{aligned}
$$

Then

$$
\begin{aligned}
\sum_{m=1}^{\infty}\left(\sum_{n=1}^{\infty} \frac{a_{n}}{m+n}\right)^{p} & <\left(\frac{\pi}{\sin (\pi / p)}\right)^{p / q} \sum_{m=1}^{\infty} \sum_{n=1}^{\infty} \frac{a_{n}^{p}}{m+n}\left(\frac{n}{m}\right)^{1 / q} \\
& =\left(\frac{\pi}{\sin (\pi / p)}\right)^{p-1} \sum_{n=1}^{\infty} \omega(q, n) a_{n}^{p} .
\end{aligned}
$$

By (1.8), (2.2) is valid. The theorem is proved.

REMARK 2.2. As $a=b=0$, inequalities (2.1) and (2.2) change to (1.1) and (1.2), respectively, hence inequalities (2.1) and (2.2) are generalization and improvement of (1.1) and (1.2), respectively. 
REMARK 2.3. As $a=b=2$, inequalities (2.1) and (2.2) change to (1.11) and (3.1) in [5], respectively, hence inequalities (2.1) and (2.2) are generalization and improvement of (1.11) and (3.1) in [5], respectively.

REMARK 2.4. We give an open question: how to determine the constant $a$ such that $1 /\left(2 n^{1 / p}+(2 / a) n^{-1 / q}\right)$ is best possible.

\section{REFERENCES}

[1] M. Z. Gao, An improvement of the Hardy-Riesz extension of the Hilbert inequality, J. Math. Res. Exposition 14 (1994), no. 2, 255-259.

[2] _ A note on the Hardy-Hilbert inequality, J. Math. Anal. Appl. 204 (1996), no. 1, 346351.

[3] L. C. Hsu and Y. J. Wang, A refinement of Hilbert's double series theorem, J. Math. Res. Exposition 11 (1991), no. 1, 143-144.

[4] L. C. Xu and Y. K. Guo, Note on Hardy-Riesz's extension of Hilbert's inequality, Chinese Quart. J. Math. 6 (1991), no. 1, 75-77.

[5] B. Yang and L. Debnath, On new strengthened Hardy-Hilbert's inequality, Int. J. Math. Math. Sci. 21 (1998), no. 2, 403-408.

[6] B. C. Yang, A refinement of the general Hilbert double series theorem, J. Math. Study 29 (1996), no. 2, 64-70.

[7] B. C. Yang and M. Z. Gao, An optimal constant in the Hardy-Hilbert inequality, Adv. Math. (China) 26 (1997), no. 2, 159-164.

Lü Zhongxue: School of Science, Nanjing University of Science \& Technology, Nanjing 210094, China

Current address: Department of Basic Science of Technology College, Xuzhou Normal University, Xuzhou, Jiangsu 221011, China

E-mail address: 7vzx1@pub.xz.jsinfo.net 


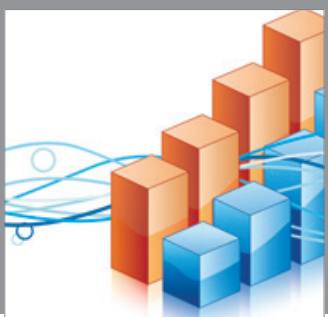

Advances in

Operations Research

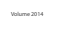

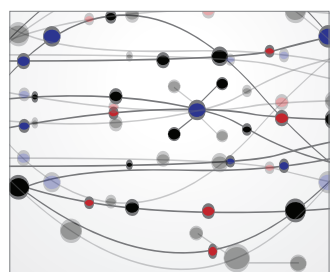

\section{The Scientific} World Journal
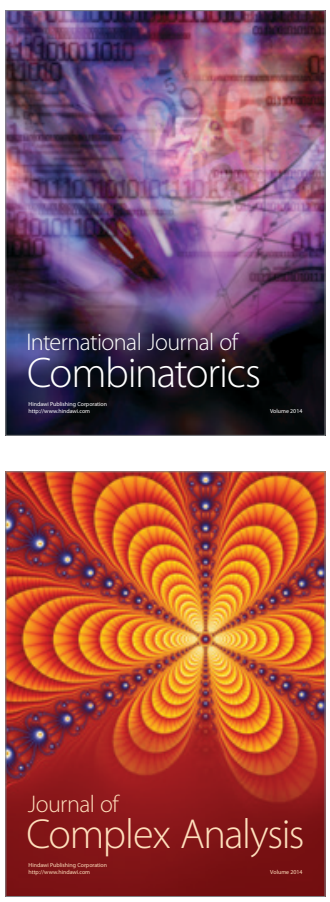

International Journal of

Mathematics and

Mathematical

Sciences
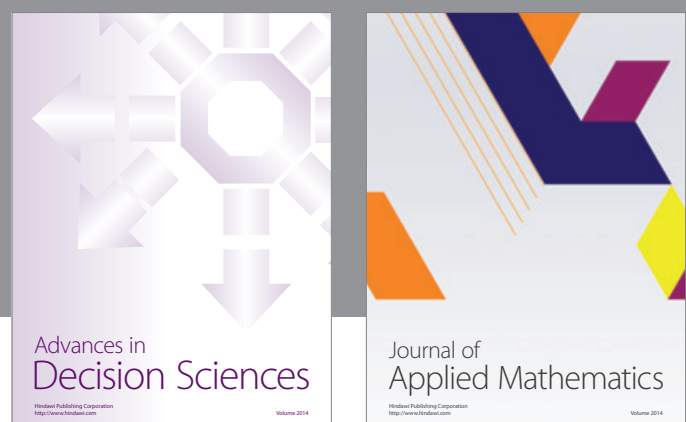

Journal of

Applied Mathematics
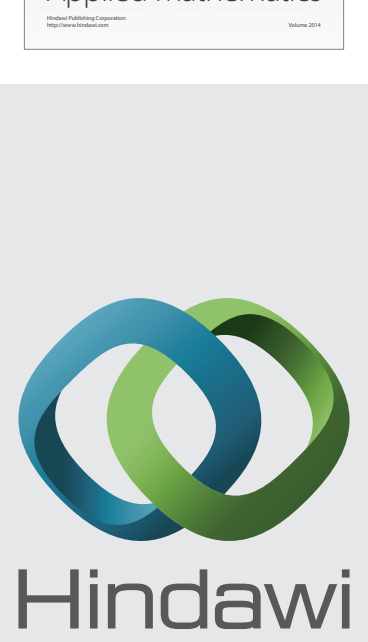

Submit your manuscripts at http://www.hindawi.com
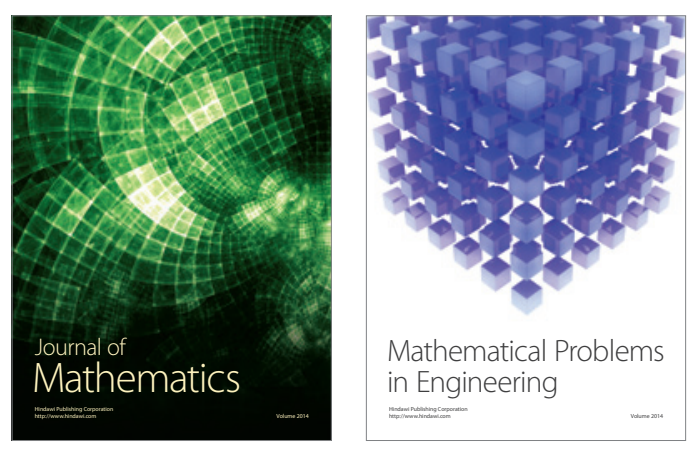

Mathematical Problems in Engineering
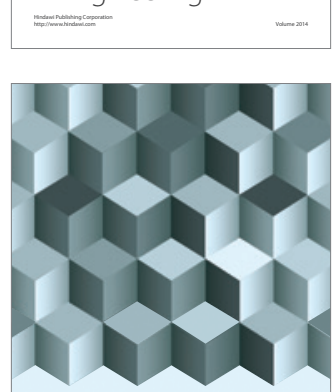

Journal of

Function Spaces
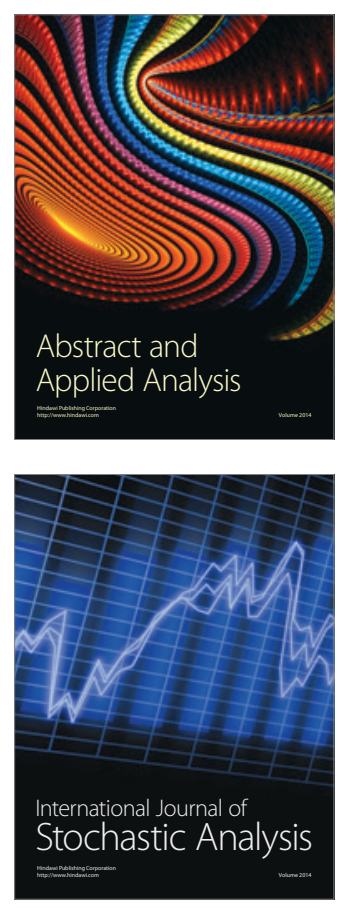

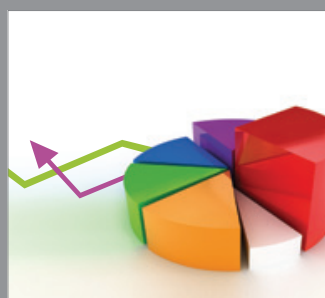

ournal of

Probability and Statistics

Promensencen
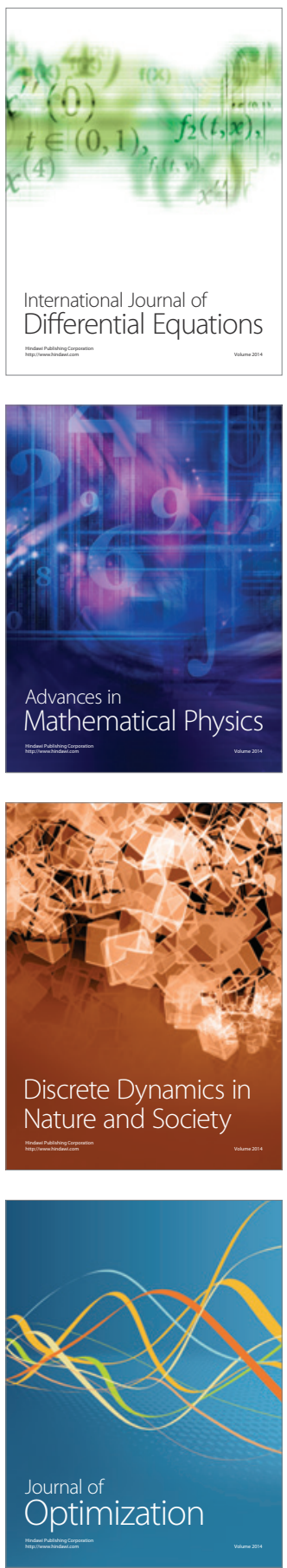